MRI-PHY/96-26, cond-mat/9610024

\title{
Calogero-Sutherland Type Models in Higher Dimensions
}

\author{
Pijush K. Ghosh* \\ The Mehta Research Institute of Mathematics 8 Mathematical Physics, \\ Allahabad-211002, INDIA.
}

\begin{abstract}
We construct two different Calogero-Sutherland type models with only two-body interactions in arbitrary dimensions. We obtain some exact wave functions, including the ground states, of these two models for arbitrary number of spinless nonrelativistic particles.
\end{abstract}

PACS numbers: 03.65.Ge, 05.30.-d

* E-mail: pijush@mri.ernet.in 
There are many exactly solvable models in one dimension, described by $N$ fermions confined in a harmonic oscillator potential or constrained to move on the rim of a circle and interacting through each other via certain special type of two-body interactions 15 . 5 . These models, namely the Calogero-Sutherland Model (CSM) and its variants, have received a renewed interest in the recent literature, because of their relevance in diverse branches of physics [6]. Unfortunately, nothing much is known in higher dimensions. This is primarily because of two reasons. Firstly, there are not many known higher dimensional models, where the fermionic description is possible for arbitrary number of particles. Secondly, the inevitable appearance of three-body interactions in the known higher dimensional analogue of CSM makes any further analysis highly nontrivial [7] 11].

The purpose of this letter is to construct higher dimensional many-body Hamiltonians with only two-body interactions, where the fermionic description of the wave functions is possible for arbitrary number of particles. With this purpose in mind, we first look for the possible ways one can construct many-body fermionic wave functions, i.e., the wave functions which are antisymmetric under the exchange of any two particle coordinates. This is also equivalent of looking for possible correlations in many-body fermionic systems. We then identify an unique correlation in arbitrary dimensions $D$ which can be realized by model systems with only two-body interactions. The correlation is such that the wave functions vanish whenever two particles are on the surface of a $S^{D-1}$ whose center coincides with the origin of the coordinate system. We construct two different models with only two-body interactions which realize such correlations. We obtain a set of exact solutions of these models. The set of exact solutions include only the bosonic ground state and some excited states for both bosons as well as fermions.

The exact wave functions of the CSM are highly correlated. These correlations are encoded in the wave functions in the form of a Jastrow factor. This Jastrow factor 
also takes care of the fermionic/bosonic nature of the wave functions. In particular, the Jastrow factor of the CSM has the form,

$$
J\left(x_{1}, x_{2}, \ldots, x_{N}\right)=\prod_{i<j} X_{i j}^{\lambda}\left|X_{i j}\right|^{\alpha}, \quad X_{i j}=x_{i}-x_{j},
$$

where $x_{i}$ denotes the particle position of the $i$ th particle. The parameters $\alpha$ and $\lambda$ are related to the strength of the inverse square two-body potential. The Jastrow factor $J$ has two interesting properties :

(a) $J$ vanishes when the position vectors of any two particles coincide. This ensures that no two particles can occupy the same position at the same time. Also, the two-body interaction in the Hamiltonian has singularities, precisely at these points, i.e., at $x_{i}=x_{j}$. (b) $J$ picks up a factor $(-1)^{\lambda}$ under the exchange of particle indices. Consequently, spinless bosons as well as fermions can be described by putting $\lambda$ equal to zero or one, respectively.

These two properties will be our basic criteria in constructing higher dimensional analogue of (11). The only freedom we have is the modification of the property (a) to accommodate more zeroes in $J$, other than those corresponding to the coinciding position vectors [9, 10]. We would expect that the zeroes of $J$ will show up as the singularities of the many-body Hamiltonian which realizes such correlations. In particular, the two-body and the three-body interactions in the corresponding Hamiltonian have the forms,

$$
\begin{aligned}
& W_{2}=\frac{g}{2} \sum_{i \neq j}\left[\vec{\nabla}_{i} \cdot \vec{\phi}\left(\vec{r}_{i}, \vec{r}_{j}\right)+g \vec{\phi}\left(\vec{r}_{i}, \vec{r}_{j}\right) \cdot \vec{\phi}\left(\vec{r}_{i}, \vec{r}_{j}\right)\right] \\
& W_{3}=\frac{g^{2}}{2} \sum_{i \neq j \neq k} \vec{\phi}\left(\vec{r}_{i}, \vec{r}_{j}\right) \cdot \vec{\phi}\left(\vec{r}_{i}, \vec{r}_{k}\right), \quad \vec{\phi}\left(\vec{r}_{i}, \vec{r}_{j}\right)=\frac{\vec{\nabla}_{i} X_{i j}}{X_{i j}}
\end{aligned}
$$

where $g=\alpha+\lambda$. The above forms of $W_{2}$ and $W_{3}$ can be obtained from the kinetic energy operator of the Hamiltonian, i.e. $-\frac{1}{2} \sum_{i} \nabla_{i}^{2} J$. $W_{3}$ vanishes for the Calogero model [1]. We will construct below higher dimensional models where $W_{3}=0$. 
Note that $X_{i j}$ in (1) is made out of the position vectors of two particles. This is because the many-body interactions encountered in physical systems can be factorised in terms of two-body interactions only. Moreover, the fermionic description for arbitrary number of particles is not possible for $X$ having more than two indices even if it satisfies the basic criteria. To see this, let us consider a (pseudo-)scalar quantity $X_{i_{1} i_{2} \ldots i_{m}}$ made out of $m(\leq N)$ position vectors such that, it picks up a minus sign under the exchange of any two indices and vanishes whenever any two indices are same. The corresponding Jastrow factor is

$$
J\left(\vec{r}_{1}, \vec{r}_{2}, \ldots, \vec{r}_{N}\right)=\prod_{i_{1}<i_{2}<\ldots<i_{m}}\left(X_{i_{1} i_{2} \ldots i_{m}}\right)^{\lambda}\left|X_{i_{1} i_{2} \ldots i_{m}}\right|^{\alpha} .
$$

Under the exchange of 'a'th and the'b'th particle, $J$ picks up a factor $(-1)^{\lambda \delta}$ with $\delta$ given by,

$$
\delta={ }^{N-2} C_{m-2}+2 \sum_{s=0}{ }^{a-1} C_{p}{ }^{b-a-1} C_{q}{ }^{N-b} C_{r}, p, q, r=0,1, \ldots, m-2, b>a,
$$

where ${ }^{N-2} C_{m-2}=\frac{(N-2) !}{(m-2) !(N-m) !}$ and $s=p+2 q+r-m+2$. The factor ${ }^{N-2} C_{m-2}$ arises from those terms where both the indices ' $\mathrm{a}$ ' and ' $\mathrm{b}$ ' are present. The second term in the right hand side of (4) receives contribution from those terms having either the ' $a$ ' or the ' $\mathrm{b}$ ' indices. The factor two comes because the contribution is same for both the cases. Thus, the second term is always even and $J$ picks up a minus sign only for odd $\delta_{1}={ }^{N-2} C_{m-2}$. This immediately implies that the fermionic nature of $J$ will be preserved for arbitrary $N$, only when $m=2$. One might also consider $X_{i_{1} i_{2} \ldots i_{m}}$ to be (pseudo-)scalar/vector and try to construct $\tilde{J}$ by taking linear combination of these [7], namely,

$$
\tilde{J}\left(\vec{r}_{1}, \vec{r}_{2}, \ldots, \vec{r}_{N}\right)=\sum_{i_{1}<i_{2}<\ldots<i_{m}} a_{i_{1} i_{2} \ldots i_{m}} X_{i_{1} i_{2} \ldots i_{m}},
$$

where $a_{i_{1} i_{2} \ldots i_{m}}$ are the suitably chosen coefficients. Unfortunately, $\tilde{J}$ has a definite symmetry under the permutation of any two particles for $N=m+1$ only. Thus, the natural 
way to describe fermionic wave functions for arbitrary number of particles is to construct Jastrow factor (11) with $X$ having only two indices.

The most general form of $X_{i j}$ in one dimension is $X_{i j}=\left(x_{i}^{k}-x_{j}^{k}\right) S_{i j}\left(x_{i}, x_{j}\right)$, where $k$ is a positive integer and $S_{i j}$ is an arbitrary function with the property that it is symmetric under the exchange of ' $\mathrm{i}$ ' and ' $\mathrm{j}$ '. Note that any odd function with $\left(x_{i}^{k}-x_{j}^{k}\right) S_{i j}$ as its argument is also a good candidate for $X_{i j}$. We will restrict ourselves throughout this letter to the polynomial form of $X_{i j}$ only. The case $k=1$ and $S_{i j}=1$ correspond to the Jastrow factor of the model considered in Ref. [1]. The trivial higher dimensional ( $D \geq 2$ ) generalization of this is $X_{i j}=\left(\left|\vec{r}_{i}\right|^{\beta}-\left|\vec{r}_{j}\right|^{\beta}\right) S_{i j}\left(\vec{r}_{i}, \vec{r}_{j}\right)$, where $\beta$ is a real positive constant. In $D \geq 3$ dimensions, this is the only possibility satisfying all the requirements discussed above as long as we are interested in constructing $X_{i j}$ solely out of the position vectors. It might be noted at this point that one can construct many different $X_{i j}$ using the Cartesian components of the position vectors. Most of these expressions for $X_{i j}$ can not be expressed solely in terms of the position vectors and even if such a reduction is possible for certain special cases, we will get back the expression mentioned above. In other words, this is the unique choice for (pseudo-)scalar Jastrow factor maintaining the basic criteria. Since we are interested in constructing model Hamiltonians, we consider $X_{i j}=\left|\vec{r}_{i}\right|^{\beta}-\left|\vec{r}_{j}\right|^{\beta}$ without loss of any generality. Now note that for $\beta=1$ and $\beta=2$, the corresponding three-body interaction terms $W_{3}(\beta=1)$ and $W_{3}(\beta=2)$ vanish, i.e.,

$$
\begin{gathered}
W_{3}(\beta=1)=\frac{g^{2}}{2} \sum_{i \neq j \neq l} \frac{1}{\left(\left|\vec{r}_{i}\right|-\left|\vec{r}_{j}\right|\right)\left(\left|\vec{r}_{i}\right|-\left|\vec{r}_{l}\right|\right)}=0, \\
W_{3}(\beta=2)=2 g^{2} \sum_{i \neq j \neq l} \frac{\vec{r}_{l}^{2}}{\left(\vec{r}_{l}^{2}-\vec{r}_{j}^{2}\right)\left(\vec{r}_{l}^{2}-\vec{r}_{i}^{2}\right)}=0 .
\end{gathered}
$$

The identity (6) is well known [1]. To prove the identity (7), first note that $W_{3}(\beta=2)$ can be conveniently written as, 


$$
W_{3}(\beta=2)=g^{2} \sum_{i \neq j \neq l} \frac{\vec{r}_{i}^{2}+\vec{r}_{j}^{2}}{\left(\vec{r}_{l}^{2}-\vec{r}_{j}^{2}\right)\left(\vec{r}_{l}^{2}-\vec{r}_{i}^{2}\right)} .
$$

Now exactly following the analysis of Ref. [1] for the corresponding term in the CSM, it can be shown that $W_{3}(\beta=2)$ vanishes.

We will construct below two different many-body Hamiltonians in arbitrary dimensions, where these two Jastrow factors appear in the corresponding wave functions. Before that, let us discuss on $D=2$ dimensions, where there are more freedom in constructing $X_{i j}$. In terms of the complex coordinates $z_{i}=x_{i}+i y_{i}$ and $\bar{z}_{i}=x_{i}-i y_{i}$, the possible $X_{i j}$ 's are (i) $X_{i j}=z_{i}^{k}-z_{j}^{k}$, (ii) $X_{i j}=\left|z_{i}\right|^{\beta}-\left|z_{j}\right|^{\beta}$, and (iii) $X_{i j}=z_{i}^{k} \bar{z}_{j}^{k}-z_{j}^{k} \bar{z}_{i}^{k}$. The expression (i) with $k=1$ appears in the Laughlin's trial wave functions for the spin polarized electrons moving in an external uniform magnetic field. No explicit model realizing such correlations can be constructed since $\sum_{i} \partial_{z_{i}} \partial_{\bar{z}_{i}} J\left(z_{1}, z_{2}, \ldots, z_{N}\right)=0$ for any $k$. Note that we have freedom of multiplying the right hand side of (i), (ii) and (iii) by $S_{i j}$. In particular, one can construct model Hamiltonian for $X_{i j}=\left(z_{i}^{k}-z_{j}^{k}\right)\left|z_{i}-z_{j}\right|$. Unfortunately, the three-body interaction is non-vanishing for such a choice. The expression (ii) has been discussed above. The three-body interaction term is nonzero for the third expression of $X_{i j}$, even though it offers novel correlations [9, 10].

Let us consider the Hamiltonian,

$$
H=-\frac{1}{2} \sum_{k=1}^{N} \vec{\nabla}_{k}^{2}+\frac{1}{2} \sum_{k=1}^{N} \vec{r}_{k}^{2}+V_{1}(\beta)+V_{2}(\beta)+W_{3}(\beta),
$$

where $V_{1}(\beta), V_{2}(\beta)$ and $W_{3}(\beta)$ are given by,

$$
\begin{aligned}
& V_{1}(\beta)=\frac{\beta^{2}}{2} g(g-1) \sum_{k \neq j} \frac{\left|\vec{r}_{k}\right|^{2(\beta-1)}}{\left(\left|\vec{r}_{k}\right|^{\beta}-\left|\vec{r}_{j}\right|^{\beta}\right)^{2}}, \\
& V_{2}(\beta)=\frac{g \beta}{2}(D+\beta-2) \sum_{k \neq j} \frac{\left|\vec{r}_{k}\right|^{\beta-2}}{\left|\vec{r}_{k}\right|^{\beta}-\left|\vec{r}_{j}\right|^{\beta}}, \\
& W_{3}(\beta)=\frac{\left|\vec{r}_{i}\right|^{2(\beta-1)}}{2} g(g-1) \sum_{i \neq j \neq k} \frac{\left.\left|\vec{r}_{i}\right|^{\beta}-\left|\vec{r}_{j}\right|^{\beta}\right)\left(\left|\vec{r}_{i}\right|^{\beta}-\left|\vec{r}_{k}\right|^{\beta}\right)}{\left(\mid \vec{r}^{2}\right.} .
\end{aligned}
$$


$g$ is a dimensionless coupling constant in (10). We are working in the units $\bar{h}=m=$ $w=1$, where $h=2 \pi \bar{h}$ is the Planck's constant, $m$ is the mass of each particle and $w$ is the oscillator frequency.

The Hamiltonian (9) is defined in arbitrary $D$ dimensions. The one dimensional results can be obtained by the replacement $\left|\vec{r}_{i}\right| \rightarrow x_{i}$ and restricting $\beta$ to take only positive integer values. The three-body interaction term in the Hamiltonian vanishes for $\beta=1,2$ as mentioned previously. However, it is nonzero for any other values of $\beta$. Note that for $\beta=1, V_{2}(1)$ can be conveniently rewritten as,

$$
V_{2}(1)=-\frac{g}{4}(D-1) \sum_{k \neq j} \frac{1}{\left|\vec{r}_{k}\right|\left|\vec{r}_{j}\right|} .
$$

This term vanishes for $D=1$ and hence, the Hamiltonian (9) reduces to the one considered in [1]. For $\beta=2, V_{2}(2)$ vanishes in arbitrary dimensions because it is antisymmetric in 'i' and 'j'. The two-body interactions $V_{1}(2)$ can be alternatively written as,

$$
V_{1}(2)=\frac{g(g-1)}{2} \sum_{k \neq j}\left[\frac{1}{\left(\left|\vec{r}_{k}\right|+\left|\vec{r}_{j}\right|\right)^{2}}+\frac{1}{\left(\left|\vec{r}_{k}\right|-\left|\vec{r}_{j}\right|\right)^{2}}\right] \text {. }
$$

Note that this is related to the root structure of $D_{N}$ for $D=1$ [3]. However, for $D \geq 2$, this interpretation is not valid since the interaction is expressed in terms of the modulus of the position vectors. All the results presented below are valid for arbitrary positive $\beta$. However, we will mention only $\beta=1,2$, while referring to the Hamiltonians with only two-body interactions.

The Hamiltonian (10) can be written in terms of the creation and anhilation operators as,

$$
H=E_{0}+\frac{1}{2} \sum_{i} \vec{A}_{i}^{\dagger} \cdot \vec{A}_{i}
$$

where the ground state energy is $E_{0}=\frac{N D}{2}+\frac{g \beta}{2} N(N-1)$ and the anhilation operators are given by, 


$$
\overrightarrow{A_{i}}=-i \vec{\nabla}_{i}-i \vec{r}_{i}+\beta g i \sum_{i \neq j} \frac{\left|\vec{r}_{i}\right|^{\beta-2}}{\left|\vec{r}_{i}\right|^{\beta}-\left|\vec{r}_{j}\right|^{\beta}} \vec{r}_{i} .
$$

Note that the limit $g \rightarrow 0$ correctly reproduces the ground state energy for $N$ bosons confined in a harmonic oscillator potential. However, we do not recover the corresponding result for $N$ fermions in $D \geq 2$ in the limit $g \rightarrow 1$. In fact, $E_{0}$ with $g=1$ corresponds to an excited state of $N$ fermions for $D \geq 2$. As we vary $g$ continuously for fixed $\beta$ and $D(\geq 2), E_{0}$ interpolates between the bosonic ground state and an excited state of $N$ fermions. This is a much more general problem in higher dimensional many-body systems, like anyons in a harmonic oscillator potential or those considered in Ref. [7, 9, 10], and at present we do not have any solutions for this.

The exact wave functions of (13) are,

$$
\psi=\left.\prod_{i<j}\left(\left|\vec{r}_{i}\right|^{\beta}-\left|\vec{r}_{j}\right|^{\beta}\right)^{\lambda}|| \vec{r}_{i}\right|^{\beta}-\left.\left|\vec{r}_{j}\right|^{\beta}\right|^{\alpha} M\left(-n, E_{0}, t\right) \exp \left(-\frac{1}{2} \sum_{k} \vec{r}_{k}^{2}\right),
$$

where $n$ is an integer, $t=\sum_{i} \vec{r}_{i}^{2}$ and $M\left(-n, E_{0}, t\right)$ is the confluent hypergeometric function. The wave function vanishes whenever any two particles lie on the surface of a $S^{D-1}$ whose center coincides with the origin of the coordinate system. This automatically ensures that no two particles are at the same position at the same time. The energy eigen values corresponding to (15) are $E_{n}=E_{0}+2 n$. Note that this expression is similar to the CSM. Unfortunately, this is not the complete spectrum. It seems that a further analysis similar to the one developed in Ref. [12] in connection with the CSM is not possible for this case. At present, we are unable to find the complete spectrum.

To conclude, we have constructed two different many-body Hamiltonians in arbitrary dimensions with only two-body interactions. We obtained the exact ground states as well as some of the excited states of these Hamiltonians. We do not know of any physical system where these model Hamiltonians are relevant. However, we expect that the exact states of these models would serve as very good variational wave-functions for physically 
interesting Hamiltonians of nonrelativistic fermions. For example, the Jastrow factor,

$$
J\left(\vec{r}_{1}, \vec{r}_{2}, \ldots, \vec{r}_{N}\right)=\prod_{i<j}\left(\left|\vec{r}_{i}\right|^{2}-\left|\vec{r}_{j}\right|^{2}\right)\left|\vec{r}_{i}-\vec{r}_{j}\right|^{\gamma}
$$

is an exact eigen function of $N$ nonrelativistic fermions interacting with each other

through $\frac{1}{\left|\vec{r}_{i}-\vec{r}_{j}\right|^{2}}$ and some other physically uninteresting three-body interactions. It is reasonable to expect that a suitable choice of the symmetric functions $S_{i j}$, which has to be found numerically in general, multiplied with $J$ would eliminate the effect of these unwanted many-body interactions. Finally, we strongly believe that further analysis of these two models along the line of CSM would provide us a better understanding on the qualitative features of higher dimensional many-body systems.

\section{ACKNOWLEDGMENTS}

I would like to thank Avinash Khare for a careful reading of the manuscript and valuable comments. 


\section{REFERENCES}

[1] F. Calogero, J. Math. Phys. (N.Y.) 10 (1969) 2191; 10 (1969) 2197.

[2] B. Sutherland, J. Math. Phys.(N.Y.) 12 (1971) 246; 12 (1971) 251; Phys. Rev. A 4 (1971) 2019.

[3] M. A. Olshanetsky and A. M. Perelomov, Phys. Rep. 71 (1981) 314; 94 (1983) 6.

[4] F. D. M. Haldane, Phys. Rev. Lett. 60 (1988) 635; B. S. Shastry, ibid. 60 (1988) 639.

[5] A. P. Polychronakos, Phys. Rev. Lett. 69 (1992) 703.

[6] B. D. Simons, P. A. Lee and B. L. Altshuler, Phys. Rev. Lett. 72 (1994) 64.

[7] F. Calogero and C. Marchioro, J. Math. Phys. (N.Y.) 14 (1973) 182.

[8] R. B. Laughlin, Phys. Rev. Lett. 50 (1983) 1395.

[9] M. V. N. Murthy, R. K. Bhaduri and D. Sen, Phys. Rev. Lett. 76 (1996) 4103;

R. K. Bhaduri, A. Khare, J. Law, M. V. N. Murthy and D. Sen, IMSc/96/08/26, IP/BBSR-96/44, cond-mat/9609012, To appear in J. Phys. A: Math. Gen..

[10] P. K. Ghosh, Novel correlations in arbitrary dimensions, MRI-PHY/96-20, condmat/9607009.

[11] A. Azhari and T. T. Truong, Phys. Lett. A 223 (1996) 111.

[12] K. Vacek, A. Okiji and N. Kawakami, J. Phys. A: Math. Gen. 29 (1994) L201. 